Project 1012275

\title{
Formation and Reactivity of Biogenic Iron Minerals
}

\author{
Ferris, F. Grant \\ University of Toronto
}

\section{RESULTS TO DATE: 1. INTRODUCTION.}

In the current study we aimed to determine how the cell surface polymers, such as lipopolysaccharide (LPS) and capsular material, of a number of strains of Shewanella influenced surface proton binding behavior. An investigation of this kind is pertinent as surface proton binding site reactivity may influence a number of important eco-physiological factors. For example, proton binding sites may sequester Fe2+, as well as other metals like Ni2+, and thus inhibit the supply and metabolic reduction of Fe3+.(Parmar et al. 2001) Further understanding the cell surface reactivity of Shewanella is also important as this may influence the ability of the microorganism to adhere and interact with metal oxides and hydroxides. This study combined acid-base titration analyses to determine proton binding behavior with SDS-PAGE analysis of LPS.

\section{MATERIALS AND METHODS}

In this study we used the following strains of Shewannella spp.. S. putrefaciens (NCTC 10695), S. baltica (63), Shewanella sp. (MR-4), S. oneidensis (MR-1), S. oneidensis (DLM-7), S. amazonensis (SB2B), S. algae (OK-1) and S. algae (BrY-DL). Cultures were grown to late exponential phase ( 17 hours) in TSB liquid media at $300 \mathrm{C}$ on a rotary shaker operating at $150 \mathrm{rpm}$. A portion of the culture was then retained for Proteinase K/SDS-PAGE analysis using silver stained gels to characterize the LPS (see Korenevsky et al., 2002 for detailed methodology). The remainder of the culture was analyzed using acid-base titrations and the data modeled using the linear programming regression method (LPM; Cox et al., 1999) to characterize the proton binding behavior of the cell surface.

\section{RESULTS AND DISCUSSION}

Acid-base titration analysis revealed strains exhibited a total surface reactive site (or proton binding site) concentration of between 0.9 umoles/mg (for S. baltica 63) and 1.4 umoles/mg (for S. amazonensis SB2B). Interestingly this is a difference of over $50 \%$ between the lowest and highest site concentration, indicating that different strains of Shewanella spp. can display quite different overall surface reactivity. Clearly such differences between strains are likely to have great significance for the preferred environment and mode of life of each strain.

The LPS of Shewanella spp. forms either a rough type, which contains just the core-oligosaccharide, or a smooth type, which exhibits an O-side chain in addition to the core-oligosaccharide. As shown in Table 1, most of the strains analyzed in this study exhibited simple rough LPS, with strains OK-1 and SB2B exhibiting smooth LPS. Table 1 also records observations of additional fringe-capsular material observed by TEM in a parallel study by Korenevsky et al., 2002. Such fringe-capsular material, along with the LPS, may have a significant controlling influence over the microbe?s surface reactivity. Comparison of LPS and EPS data with total proton binding site concentration reveals some notable trends. Firstly, the two strains which exhibit the lowest total proton binding site concentration (i.e. 63 and 10695) have only rough LPS (just core-oligosaccharide) and no additional EPS (Table 1). The two ?smooth LPS? strains (OK-1 and SB2B) exhibit higher reactive site concentrations than strains 63 and 10695. This suggests that the O-side chain may contribute significantly to the reactive site density of the bacterial surface, especially in the case of SB2B which has the highest total site density and just smooth LPS, with no additional EPS. The data also indicates a correlation between observed EPS and total site density. For example, in most cases the rough LPS strains with additional capsular material exhibit higher total site concentrations than rough strains without capsular material. 
Of additional interest is the pKa values group together to create a trend across the pKa range, which represents an overall surface reactivity envelope that could be used to predict the surface reactivity of any Shewanella spp. strain. There are at least three major peaks in the pka spectra, centered on $\mathrm{pH} 5.0$ (carboxyl or phorphoryl groups), pH 7.0 (phosphoryl groups), and pH 9.0 (amino groups). Therefore, although we have observed significant differences in total reactive site concentration between strains, there is an element of uniformity in their surface reactivity characteristics which appears common to the genus as a whole (Sokolov et al. 2001).

Observations made in this study are likely to have important significances for a number of ecophysiological factors. The overall total surface reactivity and the distribution of reactive sites between core-oligosacharide, O-side chains and capsular material is likely to affect the microorganisms ability to adhere to iron oxide/hydroxide surfaces, to aid electron capture during Fe3+ respiration and to sorb favorable metals such as Fe3+ or less favorable species such as Fe2+.

\section{Table 1}

Strain LPS type Other EPS S. baltica 63 Rough None S. putrefaciens NCTC 10695 Rough None Shewanella sp. MR-4 Rough fringe/capsule S. oneidensis MR-1 Rough fringe/capsule S. algae OK-1 Smooth fringe/capsule S. oneidensis DLM-7 Rough fringe/capsule S. algae BrYDL Rough some cells have fringe $\mathrm{S}$. amazonensis SB2B Smooth None

Table 1. Summary of LPS type of each strain determined from SDS-PAGE analysis and other observations of extracellular polymeric substances (EPS) noted in a parallel study by Korenevsky et al., 2002. Note: the capsular material was found to be not highly acidic (Korenevsky et al., 2002), although this material probably still contains some reactive sites.

\section{REFERENCES}

Parmar, N., Gorby, Y.A., Beveridge, T.J., and Ferris, F.G. (2001) Formation of green rust and immobilization of nickel in response to bacterial reduction of hydrous ferric oxide. Geomicrobiol. J. 18:375-385.

Korenevsky, A.A., Vinogradov, E., Gorby, Y. and Beveridge, T. J. (2002) Characterization of the lipopolysaccharides and capsules of Shewanella spp. Applied and Environmental Microbiology. (in press).

Cox, J. S., Smith, D. S., Warren, L. A. and Ferris F. G. (1999). Characterizing heterogeneous bacterial surface functional groups using discrete affinity spectra for proton binding. Environmental Science and Technology. 33:4514-4521.

Sokolov, I., D.S. Smith, G.S. Henderson, Y.A. Gorby, and F.G. Ferris. (2001) Cell surface electrochemical heterogeneity of the Fe(III)-reducing bacteria Shewanella putrefaciens. Environmental Science and Technology 35: 341-347

DELIVERABLES: Smith, D.S. and Ferris, F.G. Specific Surface Chemical Interactions Between Hydrous Ferric Oxide and Iron Reducing Bacteria Determined using pKa Spectra. Submitted to Journal of Colloid and Interface Science (August 2002)

Phoenix, V., Korenevsky, A.A., Ferris, F.G. and Beveridge, T.J. Relationship between surface proton binding sites and lipopolysaccharide structure of 8 Shewanella strains. Oral presentation for the International Symposium on Subsurface Microbiology, Copenhagen (September 2002) 
Sokolov, I., D.S. Smith, G.S. Henderson, Y.A. Gorby, and F.G. Ferris. (2001) Cell surface electrochemical heterogeneity of the Fe(III)-reducing bacteria Shewanella putrefaciens. Environmental Science and Technology 35: 341-347

Parmar, N., Gorby, Y.A., Beveridge, T.J., and Ferris, F.G. (2001) Formation of green rust and immobilization of nickel in response to bacterial reduction of hydrous ferric oxide. Geomicrobiol. J. 18:375-385. 